\title{
Development of an Articulating Cycling Mannequin for Wind Tunnel Testing
}

\author{
Raman GARIMELLA *1,2, Siemen MOENS ${ }^{2}$, Jochen VLEUGELS ${ }^{1}$, \\ Toon HUYSMANS ${ }^{3}$, Koen BEYERS ${ }^{2}$, Stijn VERWULGEN ${ }^{1}$ \\ ${ }^{1}$ Department of Product Development, Faculty of Design Sciences, University of Antwerp, Belgium; \\ ${ }^{2}$ Voxdale bv, Belgium; \\ ${ }^{3}$ Section on Applied Ergonomics and Design, Faculty of Industrial Design Engineering, \\ Delft University of Technology, The Netherlands
}

https://doi.org/10.15221/20.11

\begin{abstract}
Gaining aerodynamic advantage is crucial in cycling, wherein drag force can account for $90 \%$ of resistance to a cyclist. The interaction of wind with the moving human and cycling equipment also contributes to drag. Testing equipment for aerodynamic properties in a wind tunnel is of interest to manufacturers in the sports industry. However, a protocol that needs to depend on the ability of a human to accurately recreate desired poses and movements multiple times may be cumbersome during testing. Additionally, a stationary mannequin does not accurately represent real-life cycling, where pedaling is a constant. Hence, a moving mannequin is suited for such applications. In this contribution, we demonstrate the development of a 3D printed articulating cycling mannequin. A cyclist was scanned using a structured light 3D scanner. Our goal was to develop an articulating mannequin with pedalling legs and a detachable upper-body to recreate two common cycling poses - time-trial and regular. In this contribution, we describe the full development of the mannequin in time trial pose. This approach outlines a low-cost method to developing mannequins for testing applications in sports.
\end{abstract}

Keywords: 3-D scanning, modelling, cycling, 3-D printing

\section{Introduction}

Gaining aerodynamic advantage is crucial both for professional and amateur cycling performance, wherein drag force can account for $90 \%$ of resistance to a road cyclist on level ground [1]. Next to the shape and pose of the cyclist, the equipment (bicycle, apparel, and accessories) and its interaction with the wind and the moving human contributes significantly to drag [2] [3].

Wind tunnels are gold standards for aerodynamic testing. Testing athletes and equipment combinations for aerodynamic properties in a wind tunnel is of prime interest to athletes, teams and manufacturers in the cycling industry [4].

Manufacturers may currently conduct wind tunnel tests:

- in the absence of a human subject. Often, bicycles or bicycle frames are tested for aerodynamic properties and their optimization. However, without evaluating its interaction with a human being, the full picture of the conditions of the end-user i.e. a professional or amateur cyclist cannot be recreated accurately.

- employing a professional cyclist: Professional cyclists are the gold standard of usability testing. Not only are they qualified to provide precise feedback due to their high sensitivity to speed and performance changes, they are also skilled at maintaining difficult poses on the bike repetitively (as is typical of a time trial race, where riders compromise comfort for aerodynamic efficiency). However, the cost of hiring a professional cyclist for testing is extremely high and can typically only be done in the off-season period of the year.

- using a mannequin: A mannequin is sufficiently representative of the human shape, provides high reliability for testing purposes, and is portable. Relying on the ability of the human to accurately recreate poses over several iterations of testing may not be a viable approach. Hence, mannequins are often used by manufacturers for testing cycling clothes, helmets, etc. Mannequins are also used to extensively understand and visualize air flow around a cyclist in environments where a human is not allowed, e.g. a laser particle image velocimetry (PIV) setup [5]-[8].

\footnotetext{
*raman@voxdale.be
} 
A 3D-printed static mannequin of a well-known elite athlete [9] has been developed in recent years. Such a mannequin is valuable for aerodynamic testing in a single pose. However, a stationary or static mannequin does not accurately represent real-life cycling. Behavior of wind around a cyclist with static legs is different from that around a cyclist with dynamic legs. This highlights the need for articulating cycling mannequins.

Moreover, such a mannequin may not be available to other researchers or commercial entities due to difficulty of access, exclusivity clauses (as the mannequin is modeled by a famous cyclist), high cost to rent, etc. Therefore, an accessible mannequin is necessary.

In this contribution, we demonstrate the engineering and development of an articulating cycling mannequin as a valuable aid in wind tunnel testing. The mannequin is 3D printed, making it low-cost. Additionally, it would be designed as a 'modular' mannequin with articulating, easily removable and replaceable parts to represent multiple poses, to enable easy transport, and assist in faster setup times during wind tunnel testing.

\section{Method}

The flowchart in Figure 1 describes the main steps in the development of the mannequin.

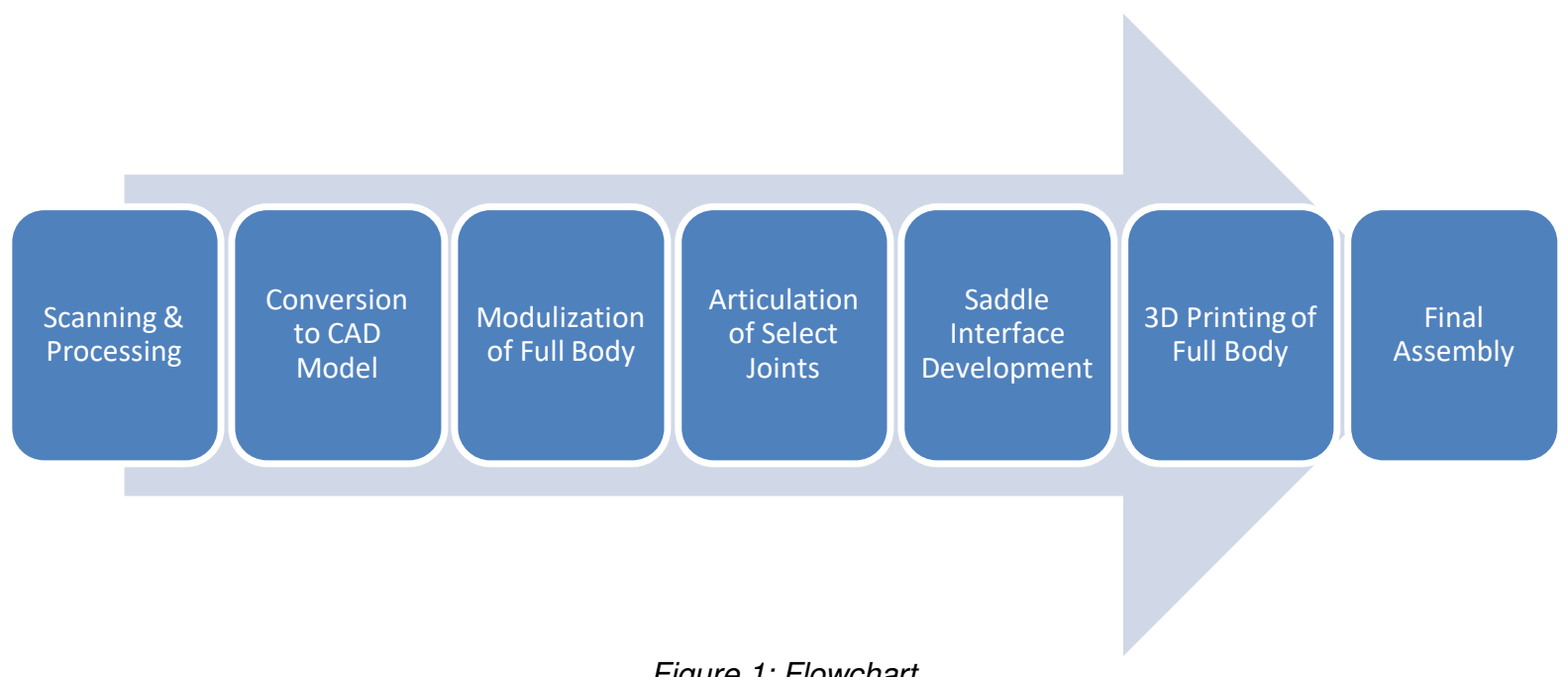

Figure 1: Flowchart

\subsection{D Scanning and Processing}

We scanned a cyclist (elite cyclist, age $=27$ years, body mass $=72 \mathrm{~kg}$, body height $=1.73 \mathrm{~m}$ ) in two poses - time trial and regular on a time trial bike and a road bike, respectively. The time trial pose (Figure 2) is an aerodynamic pose assumed by cyclists to optimize for speed. The regular pose (Figure 2 ) is where the cyclist has their hands in the dropped handlebars of the bicycle. The scans were acquired using a hand-held structured light 3-D scanner (Artec Eva, Artec Luxembourg). The duration of acquiring a scan of a cyclist is 3-4 minutes. Hence, the cyclist had to be stationary for the duration of every scan. Three scans were conducted in each pose, and the scan with the highest quality was chosen for development. The cyclist was wearing professional cycling clothes, shoes, and a swimming cap (because fine hair is not registered by the scanner). The scan was acquired at a rate of $12 \mathrm{~Hz}$. Using the scanner software (Artec Studio 14, Artec, Luxemburg), the scans were refined, artefacts were removed, and the resolution was set roughly 10,000 points in the point cloud model (.stl file type). 

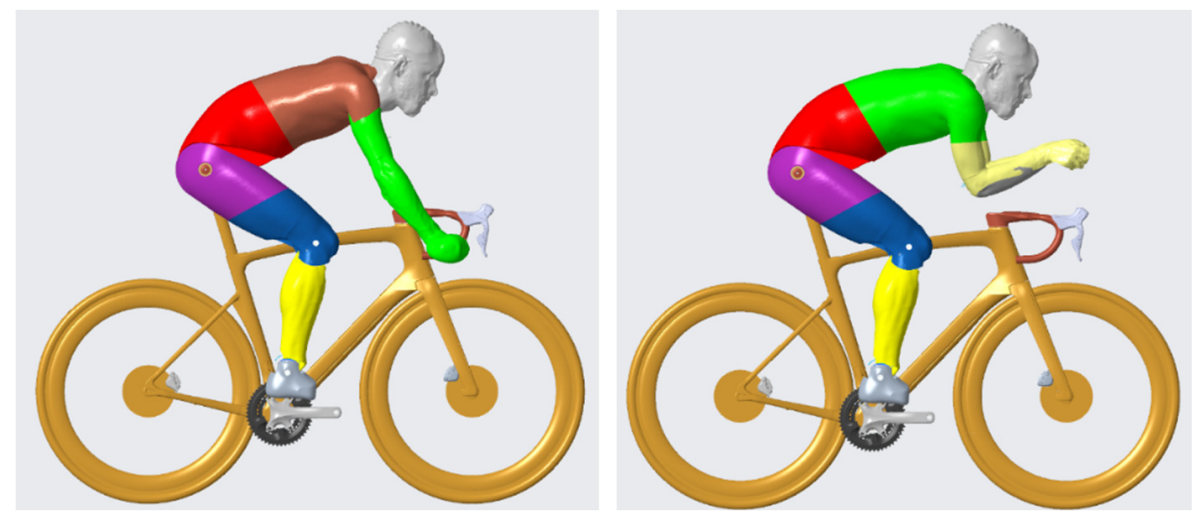

Figure 2: (Left) Regular and (right) Time Trial pose.

Note: The model was scanned in Time Trial pose on a time trial bike. This 3D illustration is of the model mounted on the same bicycle, for direct comparison of the modularity described in Section 2.2. The lower torso (in red) remains constant between the time trial and regular mode. The arms and upper torso can be changed depending on the desired pose.

\subsection{Conversion to a solid 3-D model}

The result from the previous step is a 3D file of the full body of the cyclist. Although this representation is completely watertight i.e. without any holes or gaps in the surface, it is merely a surface representation by a point cloud (Figure 3). To further make changes to this model, conversion to a solid or CAD (Computer Aided Design) model is required.
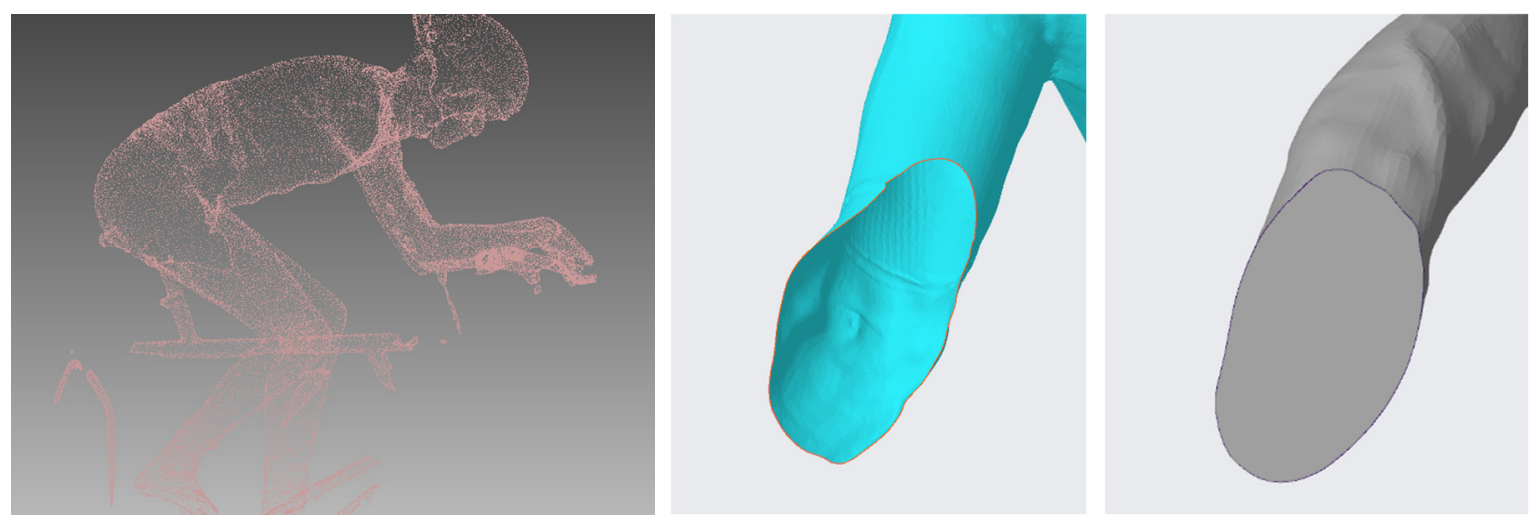

Figure 3: (Left) example of a point cloud representation from the 3D scan. (Middle and Right) Conversion to STEP file before and after

The body was segregated into subdivisions (Fusion360, Autodesk, Inc., USA), with each new part, exported as a separate STEP file (Standard for the Exchange of Product, ISO 10303-21). The division is described in the following section.

\subsection{Modulization of Full Body}

As we wanted the legs of the mannequin to be able to make a pedaling movement, the legs were cut into three segments as shown in Figure 2. In addition, we wanted to add more functionalities to the mannequin: easy to install, transport, and put clothes on. Having the possibility to remove the lower leg section and the arms will facilitate easy removal and installation of cycling clothes and make the transport volume smaller. Interfaces between these segments needed to be conceptualized.

The same type of interface was placed above the waist. The exact location was chosen so that the hip/waist part can be kept in place while other upper bodies (time trail and regular poses in our case) can interchangeably be installed.

By using a set of magnets in combination with positioning bolts, we created an interface (Figure 4) that was strong in the pedaling direction yet easy to disassemble when tilting or twisting the leg, body, or arms sideways. This sufficiently met the requirement of quick assembly/disassembly. 

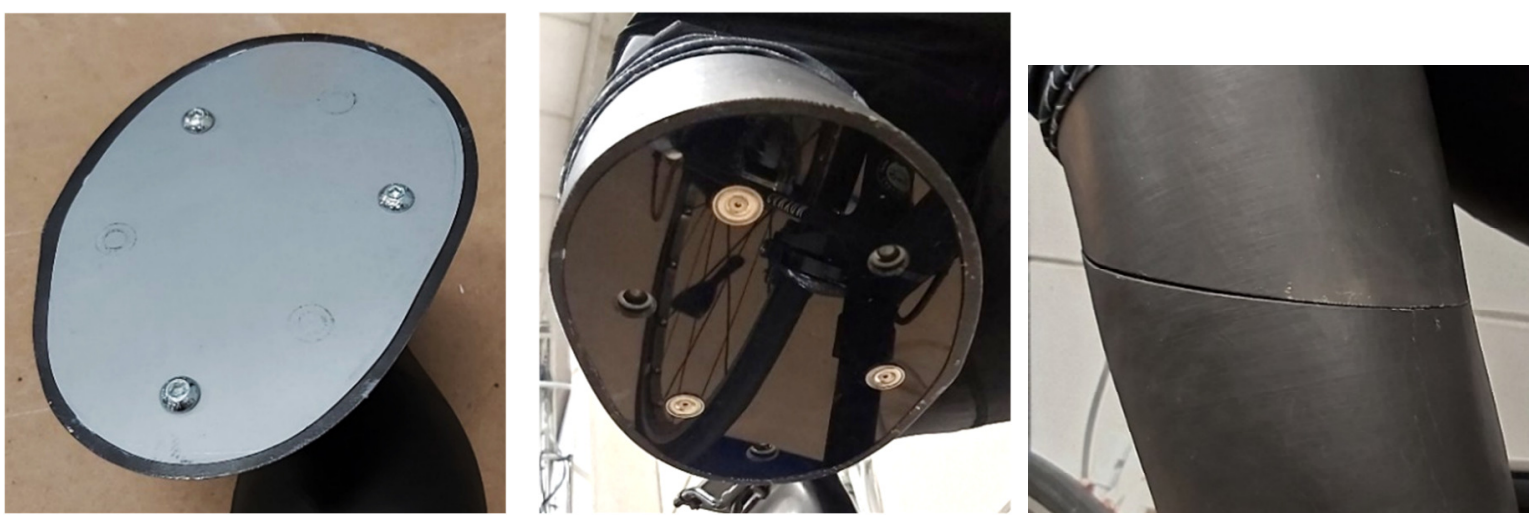

Figure 4: The magnetic interface in the upper leg portion. A similar interface was introduced for the upper body.

\subsection{Articulation of Select Joints}

This mannequin must mimic the steady movement of pedaling legs through the $360^{\circ}$ pedal stroke. The joints were not designed as 3D joints as found in healthy human beings. Instead, the angles chosen here were modeled to have degree of freedom in flexion only i.e. internal/external rotation or adduction/abduction were not part of this mannequin's joints' range of motion.

Furthermore, unlike soft tissue, the 3D printed mannequin would be rigid. Hence, cutouts had to be made to have no interference when the legs are moving. All 3 hinges (on one side of the body) have a certain degree of freedom linked to the pedaling movement (Figure 5). The range of motion for the joints of this mannequin are: $65^{\circ}$ for the left and hip joints, $130^{\circ}$ for knee joints and $80^{\circ}$ for the ankle joints.

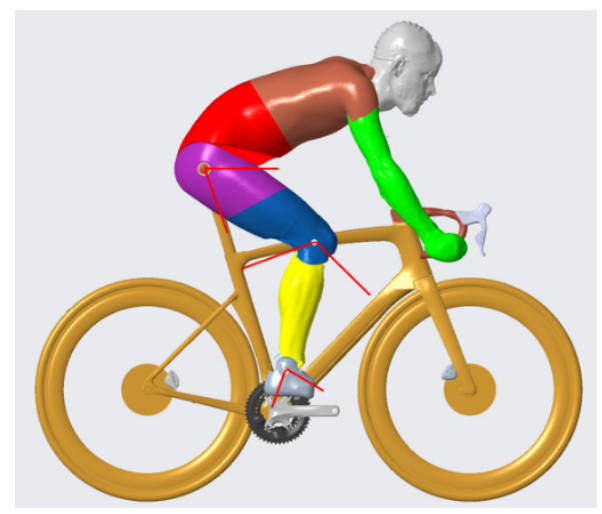

Figure 5: Range of motion for hip, knee and ankle are respectively $65^{\circ}, 130^{\circ}$ and $80^{\circ}$

These figures of range of motion were also used to determine the amount of volume around the joints that had to be cut away in the interfering parts (Figure 6).

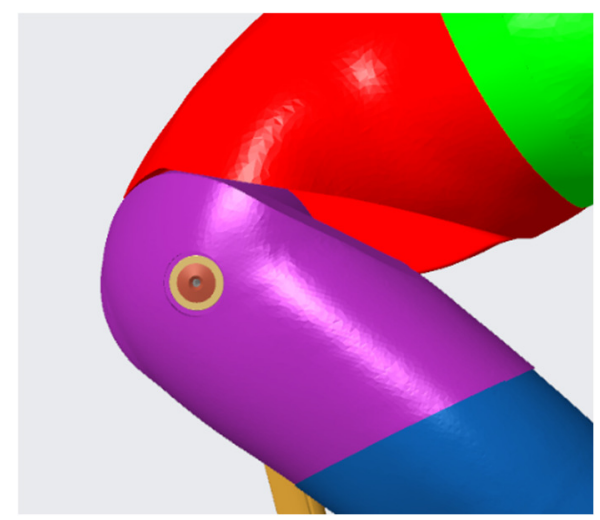

Figure 6: Figure showing degrees of freedom, and the cutout portion/clearance for left knee joint 
Since only the lower body is articulating, the hinges are located at the left and right ankle, knee, and hip. The knee and ankle have a hinge construction made from two bearings mounted in cavities in the lower leg, an axle, and a bolt/nut combination to keep the hinge together.
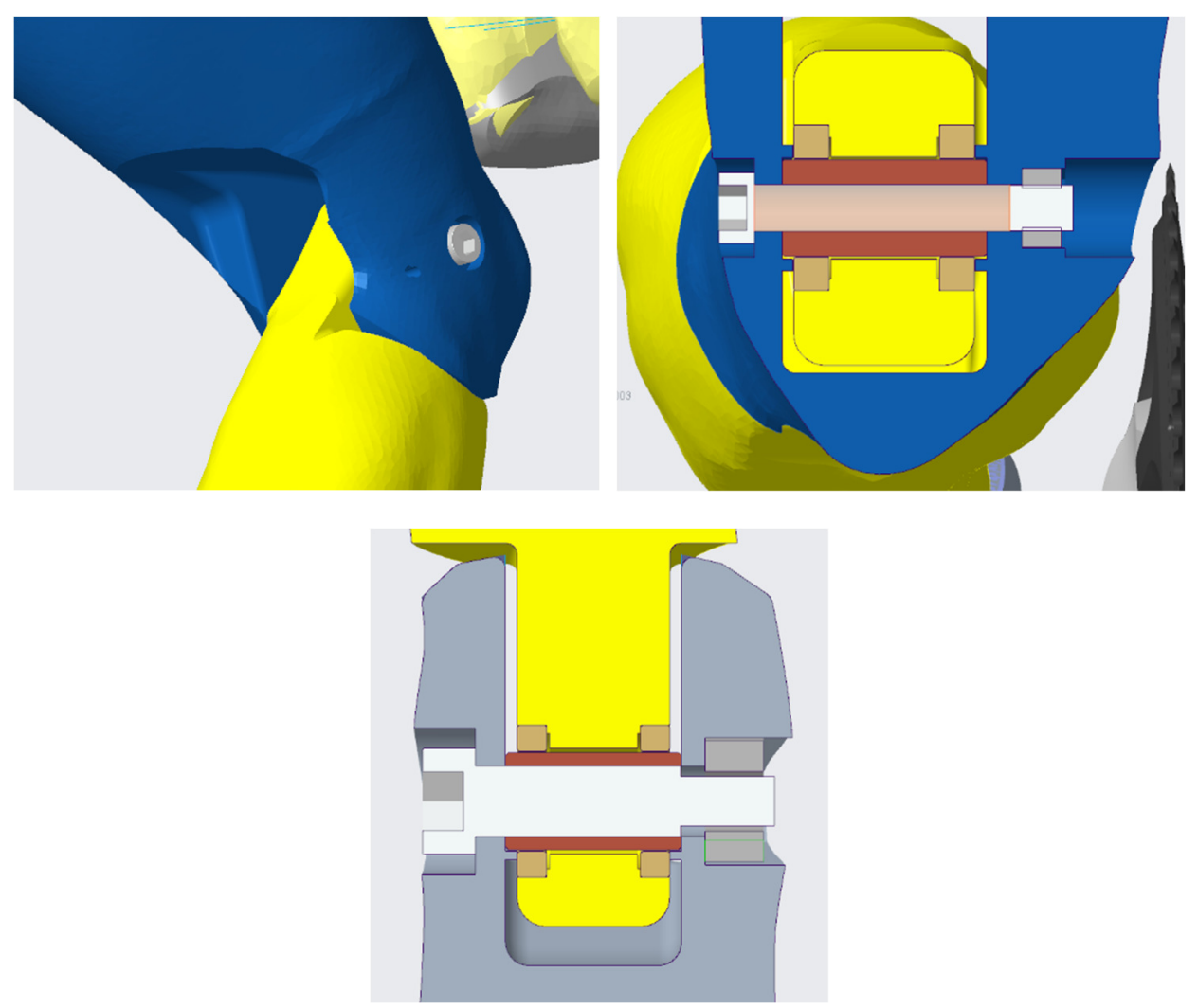

Figure 7: Knee and ankle joints. Very similar

The construction of the hip joint is different from the knee and ankle. A central steel tube was placed in the lower body, protruding to the left and right side of the body. This way the upper legs - where bearings are fitted - can be installed from each side. A bolt/washer combination is used to fix the upper legs to the steel tube, where a nut is locked on the inside of the tube.

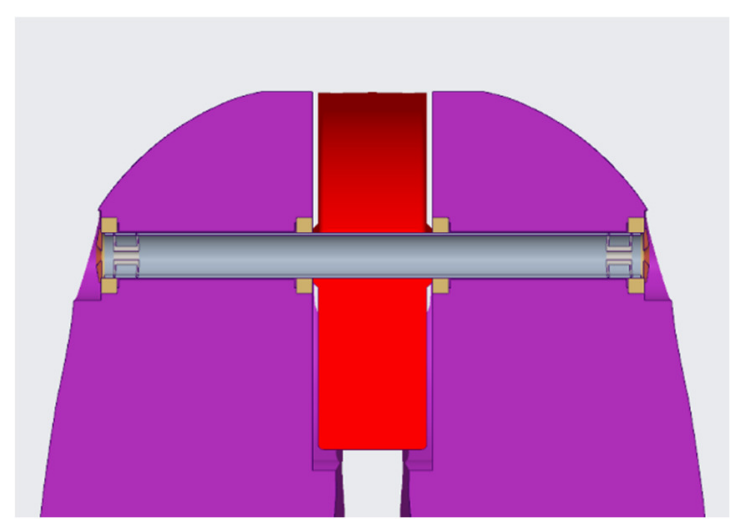

Figure 8: Hip joint construction (bottom view). The purple portions are the left and right legs.

A 'slot' was designed for the head to fit into the upper body. This slot was equipped with three metallic panels, which would serve as the fastening base for the head, which was equipped with magnets (similar to the magnets described for the saddle interface in Figure 9). Once again, the neck was modeled to have only flexion angle. The three panels provide the head with three distinct angles looking down, looking at the road, and looking ahead. Such a feature is useful for helmet testing. 


\subsection{Saddle Interface Development}

It is well known that majority of the stability of a cyclist is due to the weight borne by the rear wheel, and the saddle is the crucial point of contact to enable this weight-distribution. The mannequin is life-sized i.e. $1: 1$ scale. However, in terms of mass, the mannequin weighs about $10-15 \%$ of actual body weight. Hence, we needed to provide stability to the mannequin while it was seated on the bicycle by creating a special interface.

Bolting the mannequin to a seat post of a bicycle would be time-consuming. Moreover, as the mannequin will be wearing shorts, cutting holes in the fabric is not preferred. Hence, a magnetic interface with a custom saddle was designed. Most cycling shorts are fitted with thick padding, and to enable strong connection and stability, we mounted 8 round $20 \mathrm{~mm}$ neodymium magnets in the seat area of the mannequin. The saddle itself was replaced with a $4 \mathrm{~mm}$ steel plate connected to a standard saddle rail. This enables the mounting of this saddle on most commercially available seat posts.
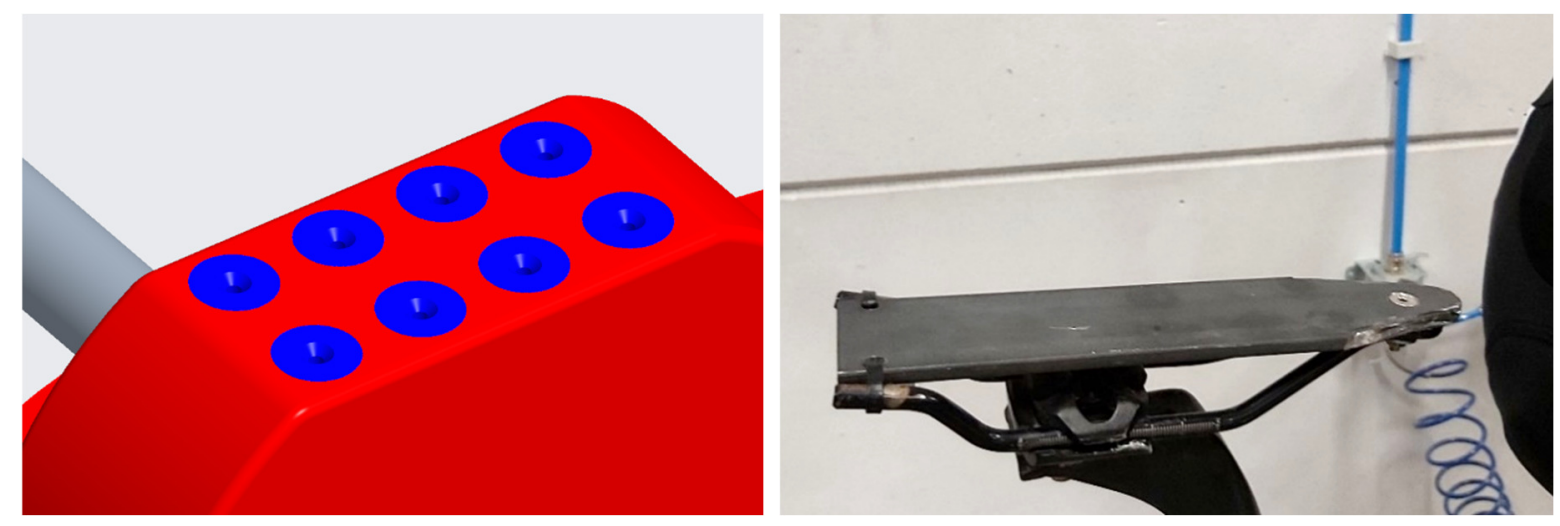

Figure 9: Saddle interface. (Left) The drawing of 8 magnets on the seat are of the mannequin. (Right) The saddle interface mounted on a standard bicycle seat post

\subsection{D Printing of Full Body}

After the concept and design phase, the files from the previous step were cleaned of any artefacts using Meshlab (ISTI-CNR, Italy) to remove inverted facets and small holes. Meshmixer (Autodesk Inc., USA) was used to smooth scanning errors and transitions.

The individual body segments as shown in Figure 2 were too large to be printed as individual entities. Hence, these segments had to be further divided and later fastened together using pins and glue. All parts were analysed to find a method of dividing such that the parts could be printed with minimal amount of support, efficient to align for re-assembly and to optimize fastening features.

All parts were printed on Prusa MK3S printers (Prusa Research a.s., Czech Republic) in PLA material (polylactic acid). The settings are as follows: $0.2 \mathrm{~mm}$ layer heights, 2 perimeters $(0.85 \mathrm{~mm}$ wall thickness) and $15 \%$ infill. For locations that house bearings (joints), local modifiers were used to provide a third perimeter for extra strength. The maximal printable size for these printers is $255 \times 210 \times 210 \mathrm{~mm}$. Parts that were split were equipped with dowel holes for pins to aid in holding the parts together during assembly.

\subsection{Final Assembly}

Apart from the pins, super-glue was used to assemble the mannequin seamlessly. The outer surface and any cracks were filled in with putty, after which all surfaces were filed using sandpaper for a smooth outer surface. Black was chosen as the colour of choice for this mannequin as it is ideal for laser-based PIV system.

\section{Results}

In total, around $9 \mathrm{~kg}$ of plastic was printed using 5 printers over a period of 5 days. Figure 10 shows the final assembly of the full body. Figure 11 shows the right knee and ankle joints in the middle of a pedal stroke i.e. not full extension. 
The mannequin works as envisioned: easily mountable on a bicycle, with complete range of motion in the pedal stroke while staying seated in the saddle.

In traditional mannequins, the limbs are rigid and offer no easy possibilities to fit clothes. Owing to the modularity, dressing the mannequin in cycling apparel was quicker by 10 times compared to a static mannequin, because arms, upper body, and legs are detachable.

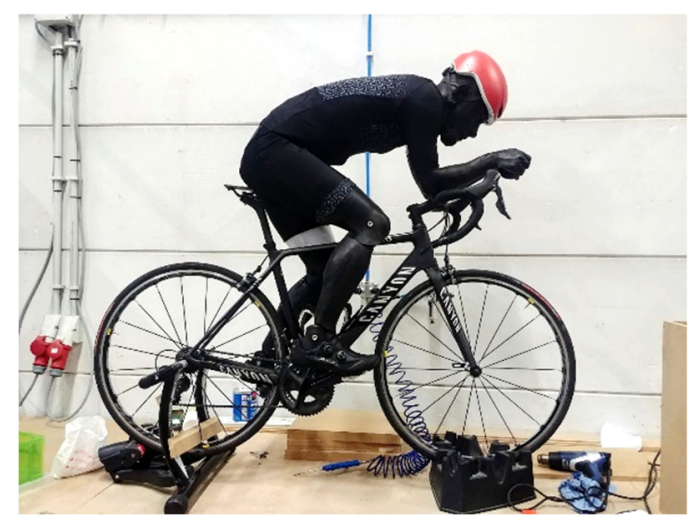

Figure 10: Mannequin mounted and ready
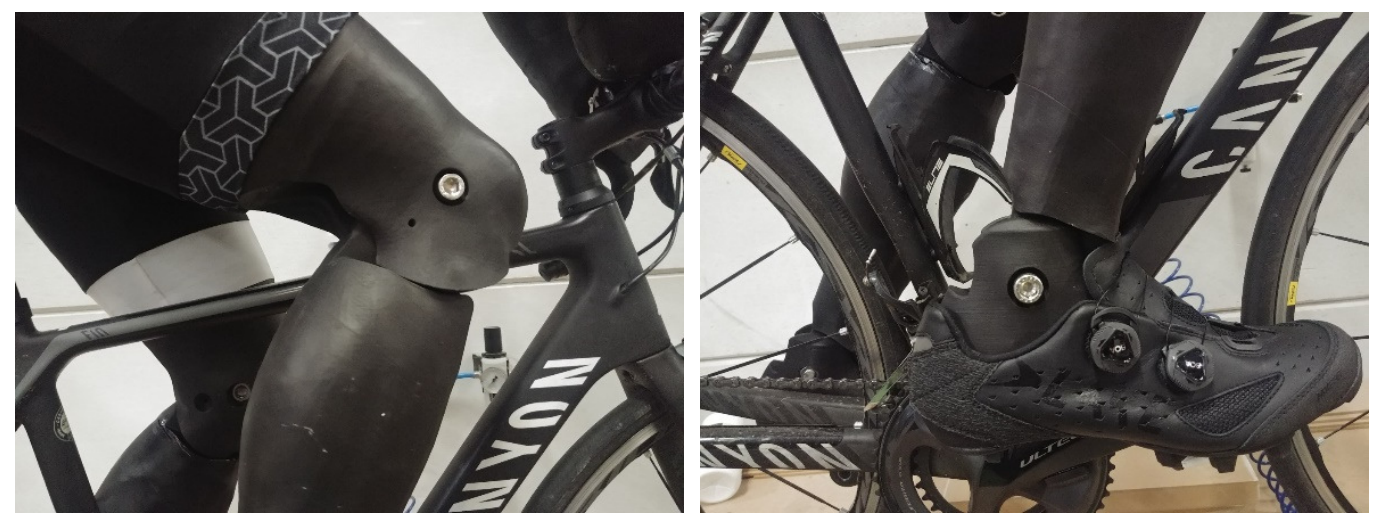

Figure 11: Knee and ankle joints side view

\section{Discussion}

The model will most likely fit on a limited range of frame sizes. Figures 10 and 11 show the mannequin mounted on a $53 \mathrm{~cm}$ bicycle frame. The parts are (re)movable but they cannot be expanded or shrunk to fit large or small bicycle frames. A 'one size fits all' design with retractable/stretchable limbs could be envisioned as future research.

The researchers note that the mannequin is not crash-resistant. This is due to the material and the thicknesses chosen during the printing process. We envision that during the usable life of such a mannequin, there will be several instances of rough handling and wear and tear, to resist which, the mannequin needs to be sturdier. However, the current material proves more than sufficient for a demonstration of the concept of articulating a mannequin for cycling purposes.

Personalized mannequins could be a potential avenue to cyclists interested in a 3D printed version of themselves. Using mathematical tools such as statistical shape modeling would make such a process easy, circumventing the need for a state of the art 3D scanner [10][11][12].

The mannequin described in this contribution functions as envisioned in principle. To test the stability of the mannequin, it needs to be positioned in a wind tunnel in high wind speed conditions $(40-65 \mathrm{~km} / \mathrm{h})$ in a range of yaw angles (i.e. crosswinds) with a motor attached to the rear wheel. In addition, the legs could be locked in various positions in the pedal stroke $\left(0^{\circ}, 90^{\circ}, 180^{\circ}\right.$ crank orientation) to test for structural integrity of the articulating legs. 


\section{Conclusion}

Construction of the mannequin described is low cost, with the material cost being the biggest contributor (under $9 \mathrm{~kg}$ for a body height of $173 \mathrm{~cm}$ ). The modularization of the body makes it easy to handle, transport and clothe the mannequin. In addition, multiple poses are represented due to this feature. Tests in a wind tunnel would further establish the validity of this mannequin as a versatile equipment testing tool (e.g. for helmets, apparel, shoes, etc.) for manufacturers, professional teams, and amateur athletes in the cycling ecosystem. As mentioned in Introduction, a mannequin also serves as the ideal surrogate for a human subject for an air-flow visualization exercise using laser techniques. An articulating mannequin would provide new insights into understanding behaviour of air around dynamic legs.

\section{Acknowledgements}

This work was supported by VLAIO (The Flemish Agency for Innovation and Entrepreneurship) under grant code "Baekeland Mandaat HBC.2016.0602". The authors would like to thank Belgian Cycling Factory and Trideus BVBA for inviting the authors to develop this project.

\section{References}

[1] J. C. Martin, D. L. Milliken, J. E. Cobb, K. L. McFadden, and A. R. Coggan, "Validation of a mathematical model for road cycling power," J. Appl. Biomech., vol. 14, no. 3, pp. 276-291, 1998, doi: 10.1123/jab.14.3.276.

[2] T. N. Crouch, D. Burton, Z. A. LaBry, and K. B. Blair, "Riding against the wind: a review of competition cycling aerodynamics," Sport. Eng., vol. 20, no. 2, pp. 81-110, 2017, doi: 10.1007/s12283-017-0234-1.

[3] A. E. Jeukendrup and J. Martin, "Improving cycling performance: How should we spend our time and money," Sport. Med., vol. 31, no. 7, pp. 559-569, 2001, doi: 10.2165/00007256-20013107000009.

[4] "Aerodynamics - Fit2fly." https://www.fit2fly.be/aero (accessed Apr. 27, 2020).

[5] L. Spurkl, L. M. Bardal, L. Sætran, and L. Oggiano, "Low aerodynamic drag suit for cycling design and testing," icSPORTS 2015 - Proc. 3rd Int. Congr. Sport Sci. Res. Technol. Support, no. icSPORTS, pp. 89-96, 2015, doi: 10.5220/0005589600890096.

[6] F. Scarano, W. Terra, and A. Sciacchitano, "Investigation of the drag crisis on the leg of a cycling mannequin by means of robotic volumetric PIV," pp. 1-9, 2019.

[7] W. Terra, A. Sciacchitano, and Y. H. Shah, "Aerodynamic drag determination of a full-scale cyclist mannequin from large-scale PTV measurements," Exp. Fluids, vol. 60, no. 2, pp. 1-11, 2019, doi: 10.1007/s00348-019-2677-6.

[8] A. D'Auteuil, G. L. Larose, and S. J. Zan, "The effect of motion on wind tunnel drag measurement for athletes," Procedia Eng., vol. 34, pp. 62-67, 2012, doi: 10.1016/j.proeng.2012.04.012.

[9] "A 3D printed mannequin of Tom Dumoulin in the TU Delft wind tunnel helps gain a competitive advantage." https://www.tudelft.nl/en/2016/tu-delft/a-3d-printed-mannequin-of-tom-dumoulin-inthe-tu-delft-wind-tunnel-helps-gain-a-competitive-advantage/ (accessed Apr. 27, 2020).

[10] T. HUYSMANS, L. Goto, J. Molenbroek, and R. Goossens, "DINED Mannequin," Tijdschrift voor Human Factors, 45 (1), pp. 4-7, Apr. 2020.

[11] A. Tsoli, N. Mahmood, and M. J. Black, "Breathing life into shape," ACM Trans. Graph., vol. 33, no. 4, pp. 1-11, 2014, doi: 10.1145/2601097.2601225.

[12] A. BALLESTER et al., "3D Human Models from 1D, 2D and 3D Inputs: Reliability and Compatibility of Body Measurements," pp. 132-141, 2018, doi: 10.15221/18.132. 